MATEC Web of Conferences 48,05004 (2016)

DOI: $10.1051 /$ matecconf $/ 20164805004$

(C) Owned by the authors, published by EDP Sciences, 2016

\title{
Development of a high-resolution apparatus to monitor physiological state of a person undergoing extreme conditions
}

\author{
Radik Kodermyatov $^{1, a}$, Maxim Ivanov ${ }^{1}$, Mikhail Yuzhakov $^{1}$, Vladimir Kuznetsov ${ }^{2}$, Mariya Yuzhakova ${ }^{1}$ \\ and Evgeniya Timofeeva ${ }^{1}$ \\ ${ }^{1}$ National Research Tomsk Polytechnic University, 634050 Tomsk, Russia \\ ${ }^{2}$ Omsk State Technical University, 644050 Omsk, Russia
}

\begin{abstract}
The present research has shown that ECG recordings and parameters of the body functional state are crucial for all kinds of the astronaut pre-flight preparations (centrifuge, thermal chamber, pressure chamber, pressure chamber with special equipment). It is, therefore, important to develop methods and tools for early detection of the preclinical forms of the functional state disorders in patients undergoing high-intensity loads of mixed character. The method based on the hardware-software complex (HSC) with nanosensors of high resolution has been proposed to measure the electrophysiological characteristics and bioelectrical impedance of the body tissues directly exposed to loading. The hardware-software complex is subjected to clinical trials in Tomsk Research Institute for Cardiology. The obtained results show that the use of nanosensors of high resolution in the HSC without standard filters allows elimination of the power-line interference in ECG recordings. Monitoring of the tissue bioimpedance parameters under test loads enables the detection of preclinical (latent) forms of various diseases. After clinical trials in Tomsk Institute for Cardiology the developed apparatus will be proposed for in-depth study of the cardiovascular system and the functional state of the body of astronauts and other persons exposed to extreme loads.
\end{abstract}

\section{Introduction}

The human body affected by various environmental factors attempts to compensate the impact of the factors, which can cause abnormalities in its organs and systems. This is due to intensification of the entire body and its tissue responses to external forces, for instance, violent changes in air pressure cause the compensatory hyperactivity of cardiovascular system to restore the state of homeostasis, that in turn leads to additional loads on other body systems - circulatory, musculoskeletal, respiratory, nervous, endocrine, etc. These processes can lead to some abnormalities in living tissues causing the resistant forms of chronic pathologies and acute forms of disorders in the organism functional state.

Early detection and prevention of abnormalities are critical for the extreme professional activities related to a long-term presence in isolated environments such as deep underwater and underground work, space flights, and others.

\footnotetext{
${ }^{\text {a }}$ Corresponding author : kodermyatovra@mail.ru
} 
The present study proposes the method based on the hardware-software complex with highly sensitive nanosensors to record biopotentials and bioelectrical impedance of living tissues in individuals exposed to extreme environments. The method allows recording abnormalities in the functional state of the tissues with high accuracy.

The study aims to investigate the effect of extreme environment on the functional activity of the human organs and tissues.

The objectives of the study are as follows: to consider the effect of extreme loads on electrophysiological characteristics of the activity of cardiovascular and musculoskeletal systems in healthy individuals; to compare the cardiovascular system parameters and the functional state of particular tissues in healthy individuals under extreme working conditions with those of having some pathologies; to propose a reliable method to monitor physiological state of individuals exposed to loads of mixed character and high intensity at the stage of initial selection, preliminary tests and simulation of extreme working conditions.

\section{Methods and research equipment}

The research equipment used in the study is a hardware-software complex with highly sensitive components for a three-lead ECG recording in the frequency range from 0 to $10000 \mathrm{~Hz}$ with the measured signals ranged from $\pm 0.3 \mu \mathrm{V}$ to $\pm 10 \mu \mathrm{V}$ and bioimpedance tissue recordings in the frequency range from 10 to $100000 \mathrm{~Hz}$ with the range of the impedance module measurement from 0.3 to $1000 \mathrm{k} \Omega$.

Those whose professional activity relates to the long-term presence in extreme environments, for instance, astronauts or deep divers, must undergo a number of stages of health monitoring, both in the preparatory period and before and during the performance of professional duties [1]. It is obvious that health assessment is crucial in the recreation and rehabilitation (in the event of temporary disability) periods due to the possible occurrence of hidden health problems in case of the medical care unavailability.

Thus, the complete and reliable diagnostic information on possible occurrence of health disorders ensure a high probability of timely preventive, therapeutic and organizational measures and, therefore, minimize the risk of emergencies in the isolated unfavorable environment.

In space medicine and labour protection, many factors affect a person working in extreme environments [2]. Most of the factors are associated with the physical effects on the body vibroacoustic, light, electromagnetic fields, ionizing and non-ionizing radiation, air environment characteristics, microclimate, and others. The combined effect of these factors, especially their extreme values, on the body triggers its reflex mobilization to preserve the state close to a healthy homeostasis, which in turn leads to the increase in loads on the corresponding systems - nervous, cardiovascular, musculoskeletal, endocrine, and others.

A significant factor of extreme physical effects on humans in spaceflight is the so-called "overload" which indicates the excess of the action of mechanical forces over the body weight. Due to the vector nature of this magnitude, overloads can be positive (action of forces is directed vertically upward from the Earth's surface) and negative (action of forces is directed towards the Earth's surface and the internal organs are "pulled down" to the opposite side regardless of the overload direction).

The overload may be long acting or shock depending on the time and intensity of exposure. The shock load occurs when the capsule is ejected from the mother ship; long-term load takes place when the spacecraft starts orbiting the Earth. 
The astronauts experience a long-term load at the liftoff and descent of the spacecraft (Figure 1). Special centrifuges are used to simulate this kind of load $[3,4,5]$.

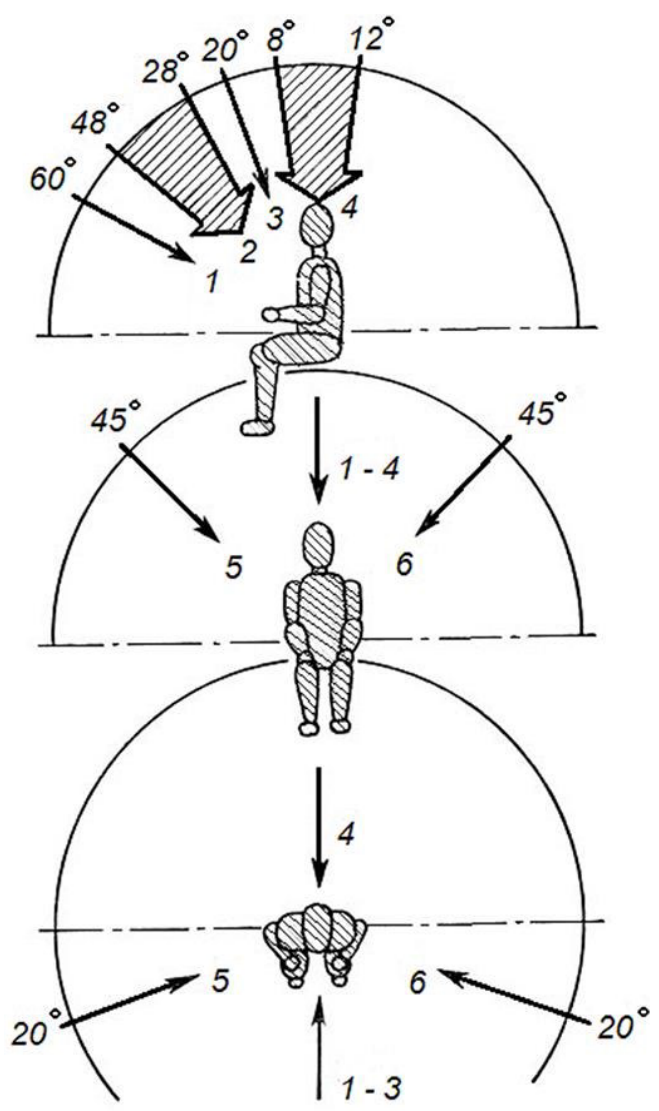

Figure 1. Schematic diagram of the overload direction relative to a seated person.

Weightless environment affects the body when the spacecraft starts orbiting the Earth and the gravity is balanced by inertial forces or almost disappears. The effect of weightlessness on the cardiovascular system results in the heart rate increase with periodical increase in pulse rate and blood pressure lowering.

The body response to weightlessness appears in sharp intensification of cardiovascular activity and hyper-compensatory reactions of muscle tissues $[3,4,5]$.

The physiological distress in case of chronic disease or other latent diseases may cause the symptom manifestation, which in turn can lead to great difficulty in providing emergency medical care in the isolated environment. Effective diagnostics at the stages of preflight preparation and assessment of the astronauts' health in the course of their professional activity will enable elimination of numerous problems associated with a "human factor" in the flight program implementation.

At the stages of preflight preparation (general space training (GST), specialized space training (SST) and preflight space training (PFST)), which total duration is from 26 to 52 weeks, general health, physical fitness, capability to endure the exposure to space environment, overload, weightlessness and other parameters are constantly monitored.

The recordings of cardiovascular system parameters are critical for all types of astronaut testing (centrifuge, thermal chamber, pressure chamber, pressure chamber with special equipment); the parameters of musculoskeletal, nervous and respiratory systems are essential for some other methods. 
The obtained information allows not only to assess the overall state of the body, but also to determine the level of preparedness and capability to endure the impact of extreme environments.

Therefore, there is an objective need to develop methods and tools for early detection of preclinical forms of diseases in individuals experiencing stresses and weightlessness. The cardiovascular diseases and diseases of spine are the most common causes for the astronaut disqualification on medical grounds. The electrophysiological methods are primarily used for the selection and examination of astronauts (electrocardiography (ECG) at rest, rheoencephalography (REG), electroencephalography (EEG), veloergometry with the dosed physical load, electromyography (EMG)).

The research group of Medical Instrument-Making Lab No. 63, Tomsk Polytechnic University, in collaboration with specialists of Omsk State Technical University have developed a hardwaresoftware complex with highly sensitive elements to record a three-lead ECG in the frequency range from 0 to $10000 \mathrm{~Hz}$ with the measured signals ranged from $\pm 0.3 \mu \mathrm{V}$ to $\pm 10 \mu \mathrm{V}$, and to record the tissue bioimpedance in the frequency range from 10 to $100000 \mathrm{~Hz}$ and the impedance module measurement range from 0.3 to $1000 \mathrm{k} \Omega$.

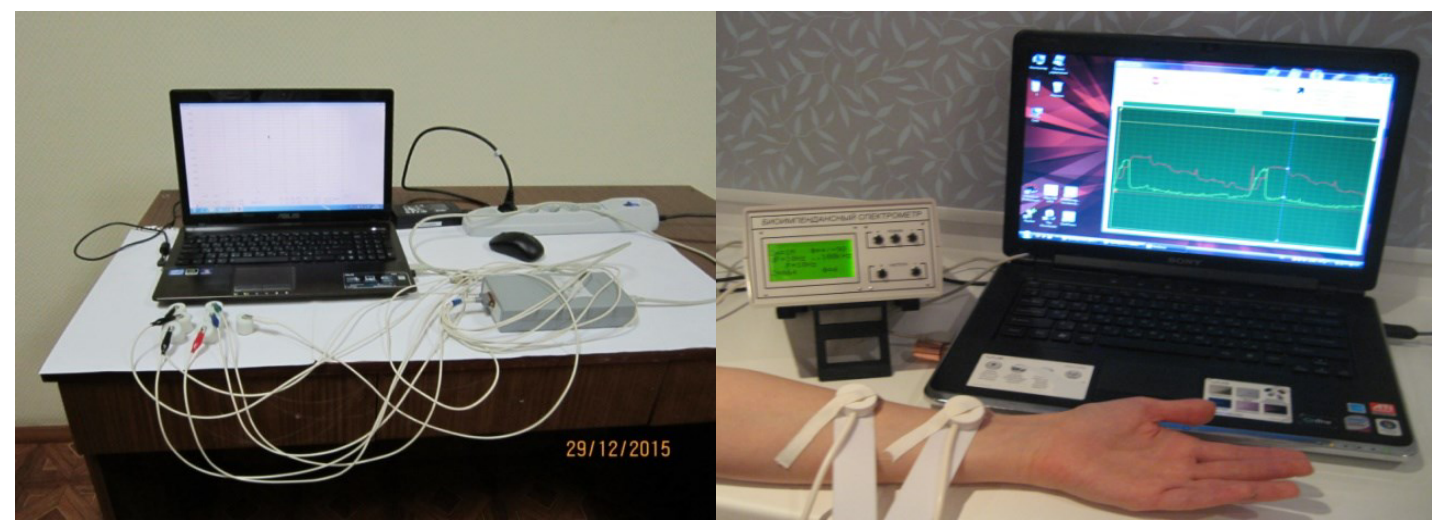

Figure 2. General view of the HSC elements (the ECG apparatus is on the left, the apparatus for bioimpedansometry is on the right).

The following are the results of the proposed HSC implementation for diagnostic studies performed in the Department of Emergency Cardiology, Tomsk Research Institute for Cardiology.

\section{Results}

A three-lead ECG measurements are regularly carried out (the frequency of medical examination is determined by the medical monitoring requirements for individuals who are allowed to work in extreme environments) without filters typical of conventional electrocardiographic equipment (high frequency filters, notch filters and tremor filters). Figure $3 \mathrm{a}, \mathrm{b}$ shows a high-resolution ECG and its spectrum recorded in a postinfarction patient in the emergency department of Tomsk Institute for Cardiology.

As can be seen from the ECG obtained without filtering, power-line interference is not found in the recorded ECG that allows reliable detection of minor changes in the patient's cardiovascular system at the early stages of pathology.

Measurements of the tissue bioimpedance are performed using the two-electrode non-invasive method by placing the electrodes on certain areas of the patient's tissue (for example, motional groups of the upper and lower limbs). The measurements are carried out in three stages: before, after and an hour after the test load on the musculoskeletal system; the results are compared with the parameters of the objective state of a person obtained by other methods. The information on the dynamics of changes is also analyzed [7]. 
Figure 4 shows the approximated spectrograms of the musculocutaneous tissue of the lower extremities in patients with different levels of physical fitness (red curve indicates the change of the impedance module and green curve indicates a phase angle): a) Group 1 (high level of physical fitness); b) Group 2 (basic level of physical fitness); c) Group 3 (unsatisfactory level of physical fitness).

The bioimpedance analysis has shown that the spectrogram of individuals with a high level of physical fitness and capable of enduring extreme environments over a long period of time differs from the spectrogram of individuals with unsatisfactory level of physical fitness in the entire frequency range (Figure 4).

a)
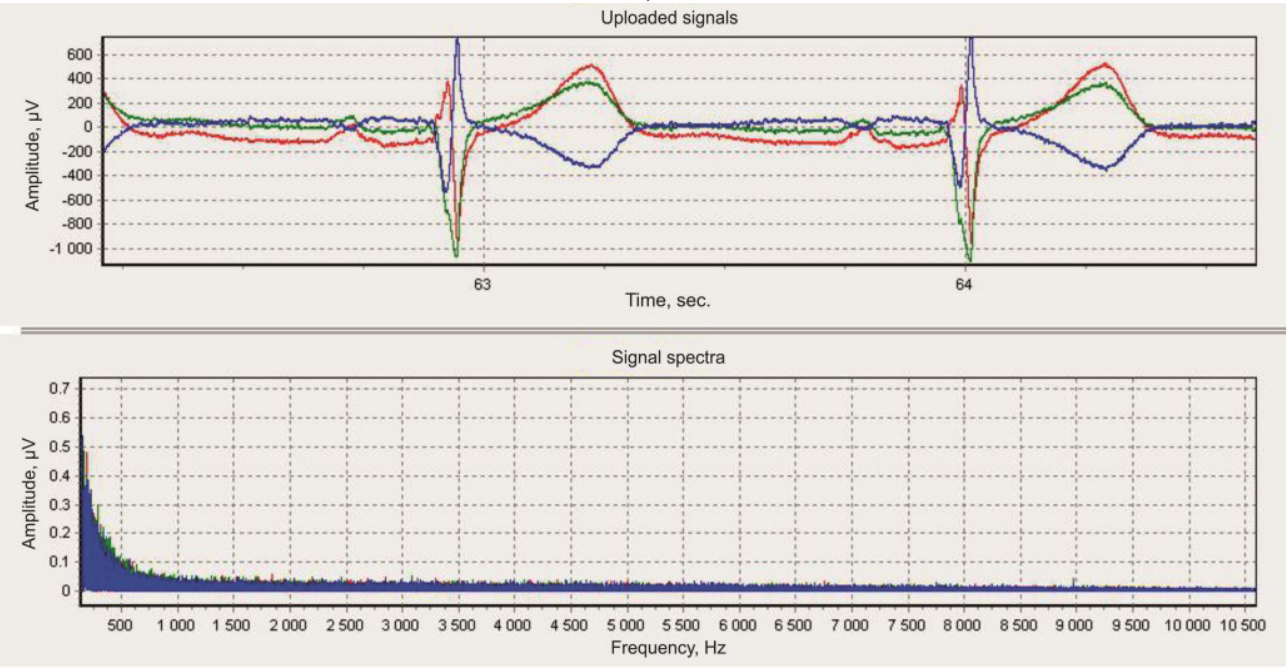

b)

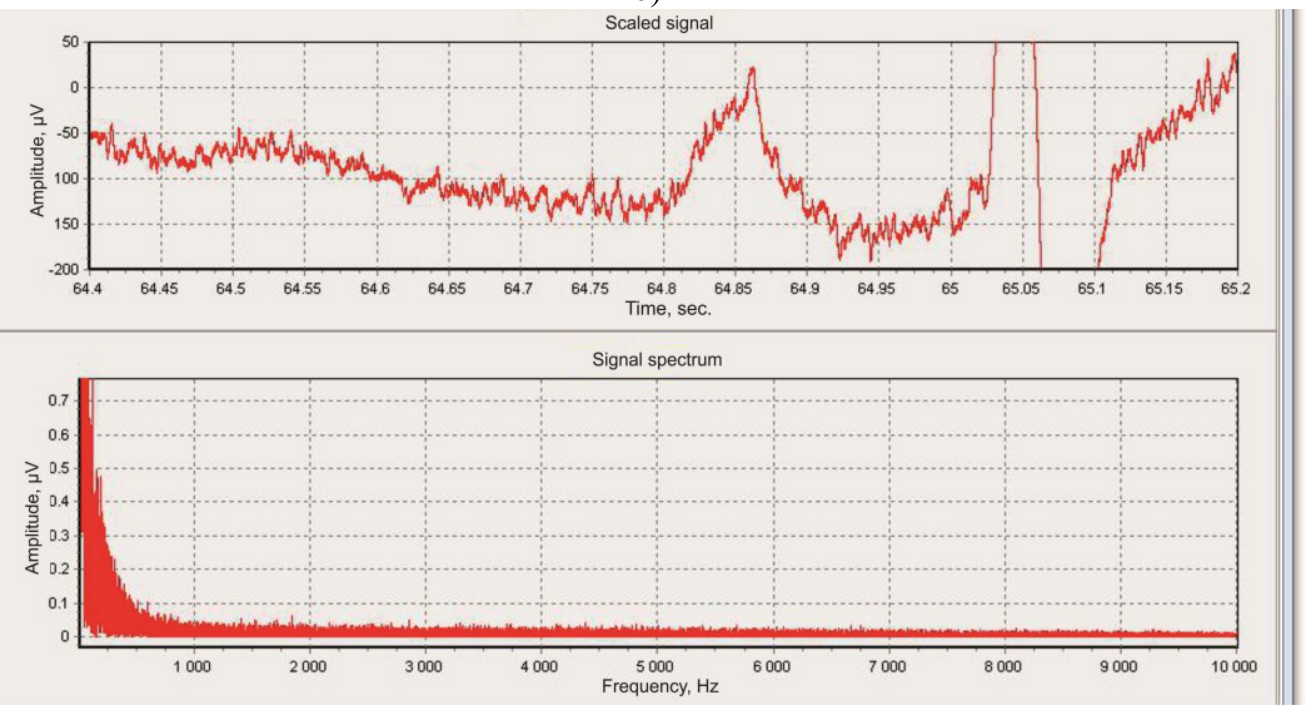

Figure 3. Patient's ECG: a) a three-lead ECG recorded from the patient's chest by the Holter method: 1 (red) - 1 lead, 2 (green) - 2 lead, 3 (blue) - 3 lead, b) ECG fragment of the 1st channel (1 lead) with the heart micropotentials at the scale sensitivity of $50 \mu \mathrm{V} / \mathrm{div}$. 
The examined individuals with no visible abnormalities in the cardiovascular system on the obtained ECG recordings experienced changes of the impedance module and its phase angle. The changes were similar to those of healthy people with a high level of physical fitness [8].

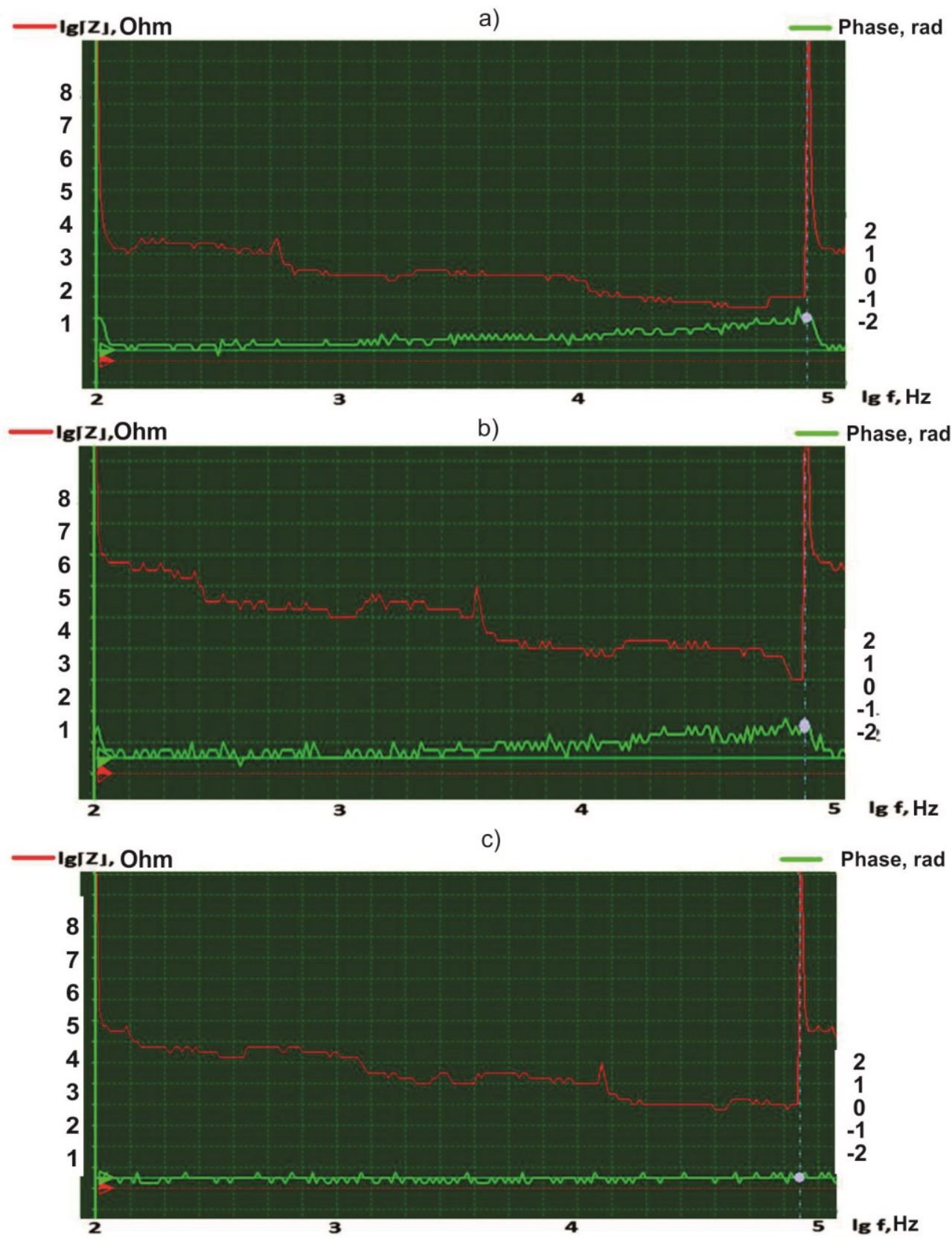

Figure 4. Approximated spectrograms of the musculocutaneous tissue of the lower extremities in patients with different levels of physical fitness (red curve indicates the change of the impedance module and green curve indicates a phase angle): a) group 1 (high level of physical fitness); b) group 2 (basic level of physical fitness); c) group 3 (unsatisfactory level of physical fitness). 


\section{Conclusion}

The present study has shown that the diagnostic information on the functional state of the body tissues and the cardiovascular system activity obtained by the developed HSC makes it possible to assess the person's capability to endure high-intensity loads of mixed character typical of space missions or underwater work. The diagnostic information helps to identify possible pathologies induced by the loads.

Further research aims to early detect the minor changes in the person's cardiovascular and musculoskeletal systems, which cannot be recorded by the common methods without highly sensitive means of obtaining diagnostic information.

After clinical trials in Tomsk Institute for Cardiology, the developed apparatus will be proposed for in-depth study of the cardiovascular system and functional state of the body of astronauts and other persons undergoing high-intensity loads of mixed character both for primary selection of astronauts and assessment of their health in the course of the professional activity.

\section{Acknowledgement}

The research was financially supported by the Federal Targeted Programme "Research and Development in Priority Fields of S\&T Complex of Russia in 2014-2020", the Agreement No. 14.578.21.0032 dated 05.06.2014 "Development of the experimental sample of a hardware-software complex for noninvasive recording of heart micropotentials in a wide frequency band without filtering and averaging in real time to early detect the symptoms of sudden cardiac death", the unique identifier of the contract: RFMEF157814X0032.

\section{References}

1. Y. Y. Xue, G. Y. Liu, B. S. Xie, X. Y. Chen, Y. Cheng, Space medicine \& medical engineering 13, $104(2000)$

2. GOST 12.0.003-74 Occupational safety standards system. Dangerous and harmful production effects. Classification

3. A. I. Grigoriev, A. R. Kotovskaya, G. A. Fomina, Acta Astronautica 68, 1495 (2011)

4. G. M. Pantalos, T. E. Bennett, M. K. Sharp, S. J. Woodruff, S. D. O'Leary, K. J. Gillars, T. Schurfranz, S. D. Everett, M. Lemon, Current Pharmaceutical Biotechnology 6, 331 (2005)

5. S. Ilyas, M. Aslam, Medical Forum Monthly 13, 1 (2002)

6. Kriterii otbora v kosmonavty i kosmicheskie turisty. Spravka. URL:

http://ria.ru/spravka/20100428/227827425.html (2016)

7. V. V. Kuznetsov, A. A. Novikov, Omsk Scientific Bulletin 2(116), 235 (2013)

8. V. V. Kuznetsov, A. A. Novikov, VI All-Russian Scientific Conference "Young Russia: advanced technology - in the industry" 3, 229 (2015) 